typeset using JPSJ.sty $<$ ver.1.0b $>$

\title{
Josephson Current Flowing in Cyclically Coupled Bose-Einstein Condensates
}

\author{
Makoto Tsubota and Kenichi Kasamatsu \\ Department of Physics, Osaka City University, Sumiyoshi 558-8585
}

(Received October 18, 2018)

\begin{abstract}
The Josephson effect in cyclically coupled Bose-Einstein condensates is studied theoretically. We analyze the simultaneous Gross-Pitaevskii equations with coupling terms between adjacent condensates. Depending on the initial relative phases between condensates, Josephson current flows cyclically to create a quantized vortex. Reducing the coupling between condensates changes the motion from periodic to chaotic, thus suppressing the cyclic current. The relation to the Kibble-Zurek mechanism is discussed; the density of the generated vortices is less than that predicted by this mechanism.
\end{abstract}

KEYWORDS: Josephson effect, Kibble-Zurek mechanism, vortex generation, Bose-Einstein condensation

The experimental observation of the Bose-Einstein condensation (BEC) in a dilute gas of neutral, trapped atoms opens up studies of interesting quantum phenomena on the macroscopic scale.14 The broken gauge symmetry due to BEC produces a macroscopic wave function (the order parameter) $\Psi(\boldsymbol{r}, t)=\sqrt{\rho} e^{\imath \theta}$, with $\rho$ as the condensate density and $\theta$ as its phase. This wave function obeys the Gross-Pitaevskii equation (GPE), which has successfully explained many phenomena of the BEC of neutral atoms. One of the significant effects caused by this wave function is the Josephson effect. Raghavan et al. theoretically studied the coherent atomic tunneling between two weakly coupled BECs 2) They analyzed the Feynman two-state model derived from the GPE to investigate the characteristic nonlinear phenomena which are not accessible with superconductor Josephson junctions.

The first motive of this work is to improve the density and phase dynamics for multicoupled BECs; this theoretical study can lead to new experiments. The second motive is closely related to the experimental observation of vortex generation in superfluid ${ }^{3} \mathrm{He}$ and ${ }^{4} \mathrm{He}$. Symmetrybreaking phase transitions in the early universe immediately after the Big Bang are expected to leave behind long-lived topologically stable structures such as monopoles, strings or domain walls. Zurek discussed the analogy of the second-order phase transition between cosmological strings and quantized vortices in the superfluid, and suggested a cryogenic experiment which tested the cosmological scenario for string formation.3) The Kibble-Zurek (KZ) scenario is the following: after the system enters the broken symmetry phase through a rapid phase transition, the ordered phases start to form, simultaneously and independently, in many parts of the system. Each ordered region has an independent phase $\theta$ of the order parameter field $\Psi(\boldsymbol{r}, t)$, because they are disconnected causally from each other. Critical slowing down near the transion temperature suppresses the velocity with which the order parameter coherence spreads. Consequently, when the disconnected domains grow together and superfluid coherence becomes established throughout the entire volume, the topological defects around which supercurrent flows, i.e., the vortices, are frozen out at the boundaries. Zurek estimated the defect density from the coherent length which is comparable to the frozen domain size. it should be noted that this estimation is based on the assumption that the boundary between more than three domains always creates a defect. This theoretical scenario mptivated various experimental works. The experiments 5 which heated small regions in superfluid ${ }^{3} \mathrm{He}$ by a neutron-induced nuclear reaction yielded results consistent with the theoretical estimation of the vortex tangle density, while Volovik subseqently noted that the experimental work tating superfluid ${ }^{3} \mathrm{He}$ evidenced not only the vortices generated by the $\mathrm{KZ}$ mechanism but alsp those induced by the rotating flow 6 ) The experiment which expanded liquid ${ }^{4} \mathrm{He}$ rapidly through the lambda line observed a density that was at least two orders of magnitude less than the theoretical estimation; the reason for the disagreement remains unresolved. Hence, at present, the $\mathrm{KZ}$ scenario is not necessarily confirmed experimentally.

Thus, this paper discusses one important factor which may be omitted in the above scenario. A cyclic supercurrent should flow through adjacent weakly coupled domains in order for a vortex to appear there. However, it is not clear whether cyclically coupled domains necessarily result in cyclic Josephson current (CJC) or not. Therefore, by improving the physics of two weakly coupled BECs, this work studies three cyclically coupled BECs. Whether CJC appears or not is found to depend on the initial relative phases and the coupling constants between adjacent BECs. It is impossible to control the initial relative phases. Hence, if initial relative phases are assumed to take arbitrary values between 0 and $2 \pi$ with equal probability, we obtain the expected value of CJC which depends on the coupling constants. By applying these results to more than three cyclically coupled 
BECs, we find that the density of generated vortices may be reduced compared with the original $\mathrm{KZ}$ scenario.

First, the formulation for the two weakly coupled BECs is described briefly.2) The macroscopic wave function $\Psi(\boldsymbol{r}, t)$ in a trap potential $V(\boldsymbol{r})$ at $T=0$ satisfies the GPE

$$
\begin{aligned}
\imath \hbar \frac{\partial \Psi(\boldsymbol{r}, t)}{\partial t}= & -\frac{\hbar^{2}}{2 m} \nabla^{2} \Psi(\boldsymbol{r}, t) \\
& +\left[V(\boldsymbol{r})+g_{0}|\Psi(\boldsymbol{r}, t)|^{2}\right] \Psi(\boldsymbol{r}, t),
\end{aligned}
$$

with $g_{0}=4 \pi \hbar^{2} a / m, m$ the atomic mass, and $a$ the $s$ wave scattering length of the atoms. When $V(\boldsymbol{r})$ is a double-well trap, $\Psi(\boldsymbol{r}, t)$ is written as

$$
\Psi(\boldsymbol{r}, t)=\psi_{1}(t) \Phi_{1}(\boldsymbol{r})+\psi_{2}(t) \Phi_{2}(\boldsymbol{r}),
$$

where $\Phi_{1,2}$ describe the condensate which is localized in each trap, $\psi_{1,2}(t)=\sqrt{N_{1,2}} e^{\imath \theta_{1,2}(t)}$ and the total number of atoms $N_{T}=N_{1}+N_{2}=\left|\psi_{1}\right|^{2}+\left|\psi_{2}\right|^{2}$. The weak coupling causes the overlap integral $\int \Phi_{1}(\boldsymbol{r}) \Phi_{2}(\boldsymbol{r}) d \boldsymbol{r}$ to be negligible, which leads to the nonlinear two-mode dynamical equations

$$
\begin{aligned}
\imath \hbar \frac{\partial \psi_{1}}{\partial t} & =\left(E_{1}^{0}+U_{1} N_{1}\right) \psi_{1}-K \psi_{2} \\
\imath \hbar \frac{\partial \psi_{2}}{\partial t} & =\left(E_{2}^{0}+U_{2} N_{2}\right) \psi_{2}-K \psi_{1} .
\end{aligned}
$$

Here, $E_{1,2}^{0}$ are the zero-point energies in each well, $U_{1,2} N_{1,2}$ the atomic self-interaction energies and $K$ the amplitude of the tunneling between condensates. Introducing the population imbalance $z(t) \equiv\left(N_{1}(t)-\right.$ $\left.N_{2}(t)\right) / N_{T}$ and the relative phase $\phi(t) \equiv \theta_{2}(t)-\theta_{1}(t)$, and rescaling to a dimensionless time $2 \mathrm{Kt} / \hbar \rightarrow t$, eqs.(3a) and (3b) are reduced to the canonical form:

$$
\frac{d z}{d t}=-\frac{\partial H}{\partial \phi}, \quad \frac{d \phi}{d t}=\frac{\partial H}{\partial z} .
$$

Here, the Hamiltonian is

$$
H=\Delta E z+\frac{\Lambda z^{2}}{2}-\sqrt{1-z^{2}} \cos \phi
$$

and the parameters are

$$
\Lambda=\frac{\left(U_{1}+U_{2}\right) N_{T}}{4 K}, \Delta E=\frac{E_{1}^{0}-E_{2}^{0}}{2 K}+\frac{\left(U_{1}-U_{2}\right) N_{T}}{4 K} .
$$

Although the exact solutions of eq. (4) are studied in detail in Ref.2, investigating the conserved energy $H$ as a function of $z(t)$ and $\phi(t)$ qualitatively reveals its dynamics. One characteristic motion is "macroscopic quantum self-trapping" (MQST); the average population difference between two BECs is nonzero. Another is $\pi$ phase oscillations which are observed experimentally in the weak link separating superfluid ${ }^{3}$ He-B reservoirs. These Josephson $\pi$ states and their quantum decay have also been studied by Hatakenaka 9 )

Extending the above formulation, we will study $n$ cyclically coupled BECs. The substitution of the representation $\Psi(\boldsymbol{r}, t)=\sum_{i} \psi_{i}(t) \Phi_{i}(\boldsymbol{r})$ into eq. (1) yields

$$
\imath \hbar \frac{\partial \psi_{i}}{\partial t}=\left(E_{i}+U_{i} N_{i}\right) \psi_{i}-K_{i, i-1} \psi_{i-1}-K_{i, i+1} \psi_{i+1},
$$

$$
(i=1,2, \cdots, n\rangle 7)
$$

where the cyclic boundary condition means $\psi_{0}=\psi_{n}$ and $\psi_{n+1}=\psi_{1}$. The parameter $K_{i, i+1}$ is the coupling constant between $i$ th and $i+1$ th BEC. The simplest case of $n=3$ is discussed. The insertion of $\psi_{i}=\sqrt{N_{i}} e^{\imath \theta}$ in eq. (7) yields

$$
\begin{aligned}
\hbar \dot{N}_{1}= & 2 K_{12} \sqrt{N_{1} N_{2}} \sin \phi_{12}-2 K_{31} \sqrt{N_{3} N_{1}} \sin \phi_{3}(8 \mathrm{a}) \\
\hbar \dot{N}_{2}= & 2 K_{23} \sqrt{N_{2} N_{3}} \sin \phi_{23}-2 K_{12} \sqrt{N_{1} N_{2}} \sin \phi_{1}(8 \mathrm{~b}) \\
\hbar \dot{N}_{3}= & \left.2 K_{31} \sqrt{N_{3} N_{1}} \sin \phi_{31}-2 K_{23} \sqrt{N_{2} N_{3}} \sin \phi_{26} 6 \mathrm{c}\right) \\
\hbar \dot{\theta}_{1}= & -E_{1}-U_{1} N_{1} \\
& +K_{12} \sqrt{\frac{N_{2}}{N_{1}}} \cos \phi_{12}+K_{31} \sqrt{\frac{N_{3}}{N_{1}}} \cos \phi_{31} \\
\hbar \dot{\theta_{2}}= & -E_{2}-U_{2} N_{2} \\
& +K_{23} \sqrt{\frac{N_{3}}{N_{2}}} \cos \phi_{23}+K_{12} \sqrt{\frac{N_{1}}{N_{2}}} \cos \phi_{12} \\
\hbar \dot{\theta_{3}}= & -E_{3}-U_{3} N_{3} \\
& +K_{31} \sqrt{\frac{N_{1}}{N_{3}}} \cos \phi_{31}+K_{23} \sqrt{\frac{N_{2}}{N_{3}}} \cos \phi_{23},
\end{aligned}
$$

where $\phi_{i j}=\theta_{i}-\theta_{j}$ is a relative phase. The Josephson supercurrent which flows from the $i$ th to the $j$ th condensate is expressed as

$$
J_{i \rightarrow j}=-\frac{2 K_{i j}}{\hbar} \sqrt{N_{i} N_{j}} \sin \phi_{i j} .
$$

Equations (8a)-(8f) conserve the total number $N_{T}=$ $N_{1}+N_{2}+N_{3}$ and the total energy

$$
E=\sum_{i}\left(E_{i} N_{i}+\frac{1}{2} U_{i} N_{i}^{2}\right)+\sum_{i<j} 2 K_{i j} \sqrt{N_{i} N_{j}} \cos \phi_{i j} .
$$

Since the wave function $\Psi(\boldsymbol{r}, t)$ is single-valued, the relative phases must satisfy

$$
\oint_{\mathcal{C}} \nabla \phi d \ell=\phi_{12}+\phi_{23}+\phi_{31}=2 \pi m
$$

where $\mathcal{C}$ is a contour that passes cyclically through the condensates and $m$ an integer. The variable $\phi_{31}$ is eliminated by eq. (11). Equations (8a)-(8f) show that the dynamics is independent of $m$. The following calculations are performed for the dimensionless variables:

$$
\frac{K t}{\hbar} \rightarrow t, \quad \frac{N_{i}}{N_{T}} \rightarrow n_{i}, \quad \frac{\hbar J_{i \rightarrow j}}{K N_{T}} \rightarrow j_{i \rightarrow j} .
$$

We will study the dynamics for $E_{1}=E_{2}=E_{3}=E^{\prime}$ and $K_{1}=K_{2}=K_{3}=K$. Then, the parameters for the dimensionless equations are $\epsilon=E^{\prime} / K$, and $\Lambda_{i}=$ $U_{i} N_{T} / K$ that represents the strength of the coupling. We assume $\Lambda_{1}=\Lambda_{2}=\Lambda_{3}=\Lambda$. Attention will be paid mainly to whether CJC flows or not. First, under $\Lambda=1$ and the fixed initial values $n_{1}(0)=n_{2}(0)=n_{3}(0)=1 / 3$, the initial phases $\phi_{12}(0)$ and $\phi_{23}(0)$ are changed. The oscillation of $j_{i \rightarrow j}$ is shown in Fig. 1. In Fig. 1(a) for $\phi_{12}(0)=\phi_{23}(0)=\pi / 8$, each current oscillates about zero; net currents flow neither clockwise nor counterclockwise. However, when the initial phases are changed (Figs. 1(b) and 1(c)), the time-averaged value of every current becomes negative, which means that CJC appears. 

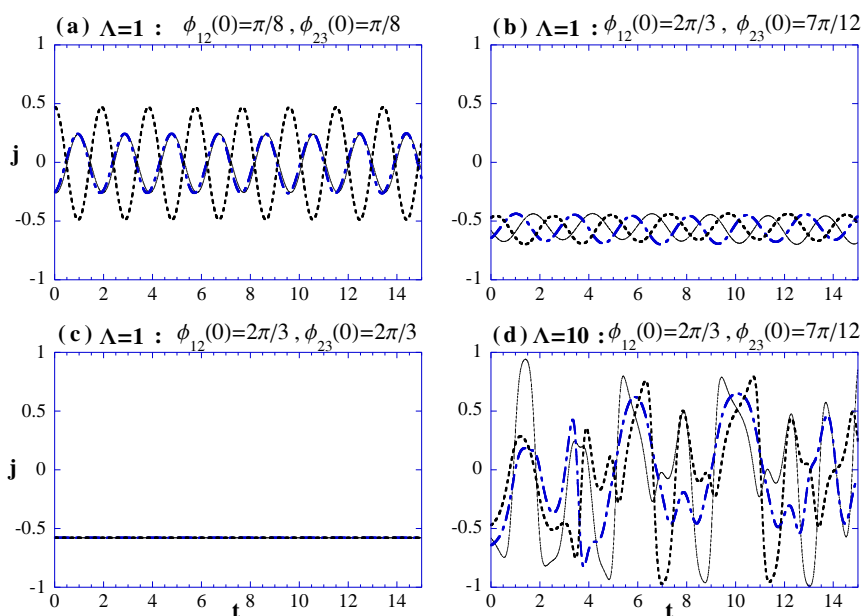

Fig. 1. Oscillation of $j_{1 \rightarrow 2}$ (solid line), $j_{2 \rightarrow 3}$ (dashed-dotted line) and $j_{3 \rightarrow 1}$ (dotted line). Values of $\Lambda$ and initial relative phases are shown in each figure.

The appearance of CJC is found by investigating the time- and piece-averaged current

$$
<j>_{a v}=\frac{1}{3 T} \int_{0}^{T} d t\left(j_{1 \rightarrow 2}+j_{2 \rightarrow 3}+j_{3 \rightarrow 1}\right),
$$

where $T$ is a period of time which is sufficiently longer than the period of oscillation.

Figure 2(a) shows how $\langle j\rangle_{a v}$ depends on $\phi_{12}(0)$ and $\phi_{23}(0)$ at $\Lambda=1$. The current $\langle j\rangle_{a v}$ has two extremums for $\phi_{12}(0)=\phi_{23}(0)=2 \pi / 3$ and $4 \pi / 3$ which give the stationary solutions. However, when $\Lambda$ is increased, i.e., the coupling is reduced, as shown in Figs. $1(\mathrm{~b})$ and 1(d), the oscillation of currents become chaotic; the transition from periodic to chaotic motion is confirmed by the analysis of the power spectrum. The important property of the $n \geq 3$ BECs dynamics is this transition to chaos which is absent in two coupled BECs.2) When the motion becomes chaotic, $\langle j\rangle_{a v}$ is lessened considerably. Thus, it is concluded that the appearance of CJC depends on the initial relative phases and the coupling constants, which are summarized in Fig. 2. Since we cannot control the initial relative phases, CJC appears with some probability that depends on the coupling constants. If every relative phase is assumed to take a value between 0 and $2 \pi$ with equal probability, the expected values or the ensemble averages of $\langle j\rangle_{a v}$ for $\Lambda=1,3$ and 10 are, respectively, $0.174,0.088$ and 0.029 . This system has some unique modes which are compared with those of two coupled BECs.2. The oscillative ones are zero-phase, $2 \pi / 3$-phase and $4 \pi / 3$-phase modes in which the time-average of the phase $\left\langle\phi_{i j}\right\rangle=0,2 \pi / 3$ and $4 \pi / 3$, respectively. There are also running-phase modes that create MQST. The analysis of the linear stability finds that the zero-phase mode is stable for an arbitrary value of $\Lambda$, while the $2 \pi / 3$ and $4 \pi / 3$-phase modes become unstable for $\Lambda>9 / 4$.

When the independent domains of the ordered phase

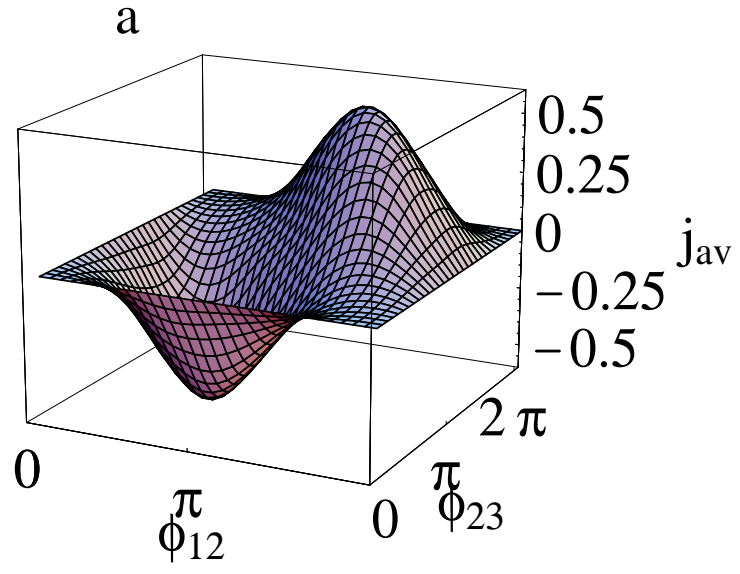

$\mathrm{b}$
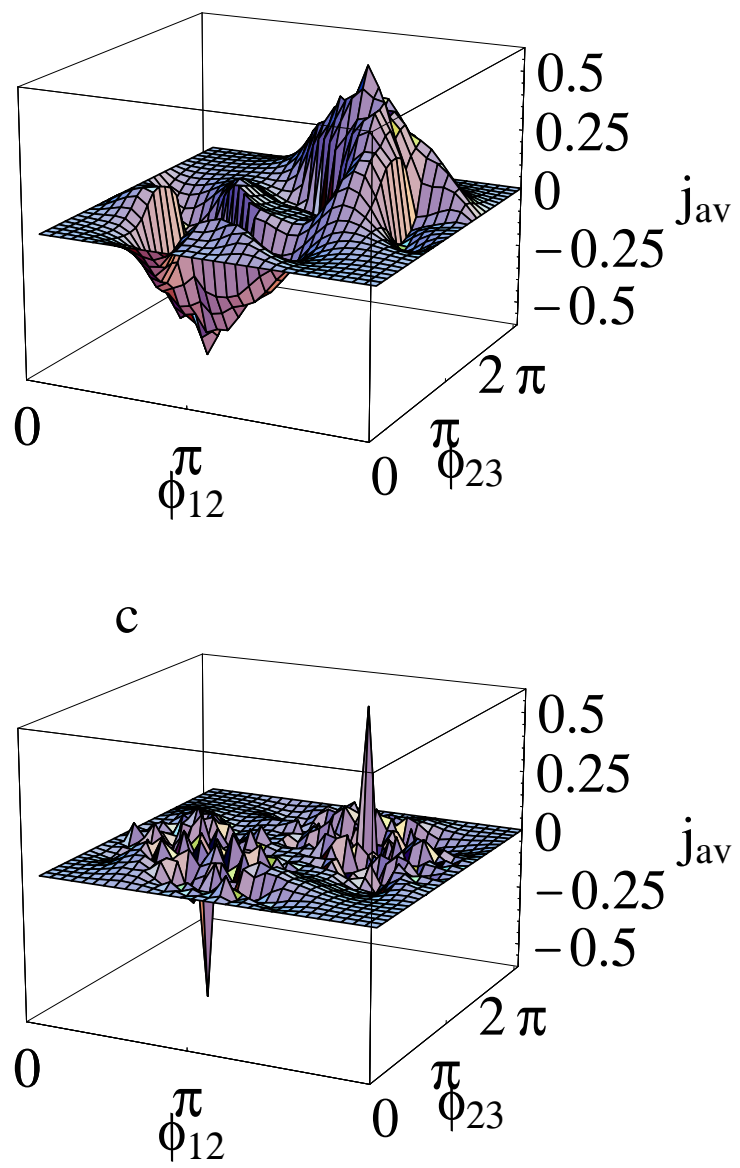

Fig. 2. Dependence of the time-averaged current $\langle j\rangle_{a v}$ on the initial relative phases $\phi_{12}(0)$ and $\phi_{23}(0)$ for (a) $\Lambda=1$, (b) 3 and (c) 10 .

grow up after the quench of the system, as described in the introduction of this paper, the coupling constants between adjacent domains are expected not to be constant but to increase with time. Such time-dependent coupling constants change the motion gradually from initially chaotic to periodic. In a real system, every pair of condensates need not have the same coupling constant $K_{i j}$. When some dissipative term is introduced to eq. 
(1), the calculation for $K_{12}>K_{23}=K_{31}$ shows that the first and second condensates are united, subsequently the united condensate and the third one create the two-body motion; the dissipative term is described in the following paper.

The above consideration is applicable to $n$ cyclically coupled BECs. This system has $2 \pi k / n$-phase modes about the stationary solutions which result from the initial relative phases $\phi_{12}(0)=\phi_{23}(0)=\cdots=\phi_{n-1, n}(0)=$ $2 \pi k / n$, where $k=0,1, \cdots, n-1$. In order to obtain the exact conclusions on CJC in $n$ BECs needs to carry out an analysis for each value of $n$. However, the following estimation for weak coupling roughly gives the dependence of CJC on $n$. Following the case of $n=3$, we will define the ensemble-averaged CJC for $n$ BECs

$$
\begin{aligned}
<j_{C J C}>_{n}=\frac{1}{(2 \pi)^{n-1}} \int\left|<j>_{a v}(\boldsymbol{x})\right| d \boldsymbol{x} \\
\boldsymbol{x} \equiv\left(\phi_{12}(0), \phi_{23}(0), \cdots, \phi_{n-1, n}(0)\right),
\end{aligned}
$$

where

$$
<j>_{a v}(\boldsymbol{x})=\frac{1}{n T} \int_{0}^{T}\left(\sum_{k=1}^{n} j_{k \rightarrow k+1}(t, \boldsymbol{x})\right) d t,
$$

is the time- and piece-averaged current starting from the initial phase $\boldsymbol{x}$. When the coupling is weak, as shown in Fig. 2(c), the main contribution for CJC comes from the stationary solutions. In the limit of weak coupling, we assume that only the stationary solutions produce CJC:

$$
<j_{C J C}>_{n}=\frac{1}{(2 \pi)^{n-1}} \sum_{k=0}^{n-1} \frac{2}{n}\left|\sin \left(\frac{2 \pi k}{n}\right)\right| .
$$

It is easy to show that $\left\langle j_{C J C}>_{n}\right.$ decreases with $n$ to disappear for $n \rightarrow \infty$. For example, we find $\left\langle j_{C J C}>_{n}=2.9 \times 10^{-2}, 4.0 \times 10^{-3}, 7.8 \times 10^{-4}\right.$ from eq. (17), respectively, for $n=3,4,5$. Even if the coupling is not weak, the relative ratio $\left\langle j_{C J C}\right\rangle_{n} /\left\langle j_{C J C}\right\rangle_{3}$ is not changed very much. When independent domains are nucleated in a system, the number of $n$ cyclically coupled BECs decreases with $n$. Hence it is concluded that CJC arises mainly from cyclic BECs with a small value of $n$. Following these results, we can estimate how the vortex density in the KZ mechanism is reduced. Then, the first assumption is that the vortices are generated only from the CJC of $n=3$ cyclic BECs based on the above considerations. The second is that the vortex density in the KZ mechanism is caused by the CJC due to the stationary solution $\phi_{12}=\phi_{23}=2 \pi / 3$, i.e., $\left.<j_{K Z}\right\rangle_{3}=0.577$ which is the maximum of $\left\langle j_{C J C}\right\rangle_{3}$, indicating that CJC flows independently of the initial relative phases. However, the CJC actually depends on the initial relative phases and the coupling constant, so that $\left\langle j_{C J C}\right\rangle_{3}$ is less than $\left\langle j_{K Z}\right\rangle_{3}$ to lessen the density of generated vortices. Figure 3 shows the dependence of $\left.<j_{C J C}\right\rangle_{3} /<j_{K Z}>_{3}$ on $\Lambda$, which can be related to the reduced ratio of the vortex density.

It is difficult to quantitatively estimate the coupling constants in superfluid helium, although it may be possible in a dilute gas of trapped atoms. More detailed

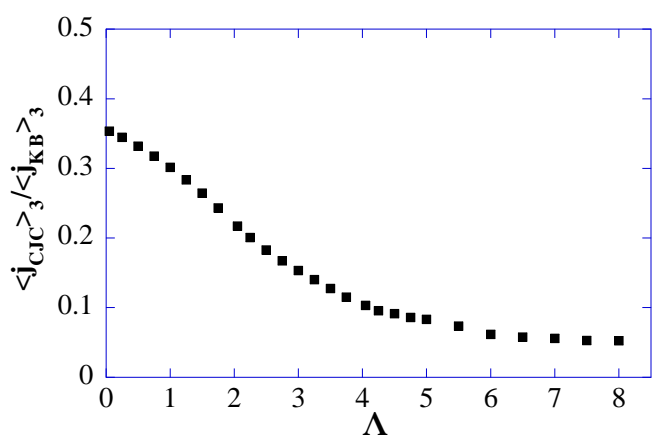

Fig. 3. Dependence of $\left.\left\langle j_{C J C}\right\rangle_{3} /<j_{K Z}\right\rangle_{3}$ on $\Lambda$.

studies that include the application to a real system and dissipative effects are to be published shortly.

The authors thank T. Iida for useful discussions.

[1] F. Dalfovo, S. Giorgini, L. P. Pitaevskii and S. Stringari: Rev. Mod. Phys. 71 (1999) 463.

[2] S. Raghavan, A. Smerzi, F. Fantoni and S. H. Shenoy: Phys. Rev. A 59 (1999) 620.

[3] W. H. Zurek: Nature 317 (1985) 505.

[4] C. Bäuerle, Yu. Bunkov, S. N. Fisher, H. Godfrin and G. R. Pickett: Nature 382 (1996) 332.

[5] V. M. H. Ruutu,V. B. Eltsov, A. J. Gill, T. W. B. Kibble, M. Krusius, Yu. G. Makhlin, B. Plaçais, G. E. Volovik and W. $\mathrm{Xu}$ : Nature 382 (1996) 334.

[6] G. E. Volovik, Physica B 280 (2000) (in press)

[7] M. E. Dodd, P. C. Hendry, N. S. Lawson, P. V. E. McClintock and C. D. H. Williams: J. Low Temp. Phys. 115 (1999) 89.

[8] S. Backhaus, S. V. Pereverzer, R. W. Simmonds, A. Loshak, J. C. Davis and R. E. Packard: Nature 392 (1998) 687.

[9] N. Hatakenaka: Phys. Rev. Lett. 81 (1998) 3753. 